\title{
COVID-19: in the uncertainty, do not try this at home
}

\author{
Tommaso Lupia $^{1}$ (1) $\cdot$ Silvia Corcione ${ }^{1,2} \cdot$ Francesco Giuseppe De Rosa $^{1}$
}

Received: 31 May 2020 / Accepted: 5 August 2020 / Published online: 17 August 2020

(C) Società Italiana di Medicina Interna (SIMI) 2020

Keywords COVID- $19 \cdot$ Pneumonia $\cdot$ SARS-CoV-2

\section{Dear Editor,}

Seventeen years after the SARS epidemic, the current SARSCoV-2 outbreak has plunged the scientific community into uncertainty once again. In December 2019, the uncertainty began with nomenclature. Initially, the new virus was called 2019-nCOV, was immediately renamed SARS-CoV-2 due to its similarities to the previous pandemic and was ultimately condensed into COVID-19.

In January 2020, this apparent instability began to involve surveillance as various public health agencies worldwide told frontline healthcare providers to be vigilant against potential imported cases using numerous approaches that differed from country to country.

Uncertainty quickly emerged at the suspected source of the contagion, the Huanan Seafood Market in Wuhan, where the virus was initially isolated, which has become a theatre of covert international schemes and conspiracy theories. Incongruities have been identified over the direct link between the Wuhan seafood market and the COVID-19 outbreak, such as by Li et al. [1], whose paper in the New England Journal of Medicine (NEJM) in late January describes a cluster of precocious laboratory-confirmed COVID-19 cases and their transmission dynamics. Of these, $45 \%$ had no direct connections to the Huanan Seafood Market, which obscures the role of this location in the outbreak.

What has occurred since then is all too clear; at the time of writing, appalling estimates point to approximately $10,400,000$ confirmed cases and 508,000 deaths, and uncertainty reigns within several machine gears, including

Tommaso Lupia

tommaso.lupia89@gmail.com

1 Department of Medical Sciences, Infectious Diseases, University of Turin, Turin, Italy

2 Tufts University School of Medicine, Boston, USA diagnostic, preventive and therapeutic approaches for COVID-19.

The 7 months into the pandemic have not yet allowed for clarifying which test could potentially be used as a gold standard for the screening, diagnosis and monitoring of COVID-19 subjects. The real-time polymerase chain reaction (RT-PCR) is able to amplify the genetic material of SARS-CoV-2, although it is possible to miss patients who have cleared the virus and have recovered from the disease. Moreover, the sensitivity of the RT-PCR swab is operator dependent and is affected by the volatility of the virus' distribution across the respiratory tract.

On the other hand, common tests, such as antibody lateral flow immunoassays and ELISA antigens, are new promising technologies but are characterised by an accuracy yet to be defined. Antibodies determine whether a patient has at some point been infected with SARS-CoV-2 and whether further testing is needed to clarify the presence of an active coronavirus infection. At this time, each test type has its own distinct advantages and disadvantages that are not fully known, and the aim is to standardise the most appropriate diagnostic algorithm to control the pandemic spread of COVID-19.

Similarly, preventive approaches are still discussed daily and enriched by new evidence, which is sometimes conflicting. A recent example of confusion in the scientific community is a published work by Zhang et al. [2] (and which included among its authors a Nobel Prize winner) in June regarding the routes of transmission and the effectiveness of mask wearing with respect to the ineffectiveness of other non-pharmaceutical interventions. This paper asserts, again after 7 months from the start, that in a world forced to social distance and quarantine, airborne transmission is the dominant route for the COVID-19 spread, although quarantine and isolation were not in place in most parts of the world (i.e. Sweden). This work has created dismay in the scientific community for its claims, but sometimes in medicine there is no absolute certainty, but only a more current certainty. 
Regarding therapeutic approaches, we learned in the first SARS crisis that supportive care would be the mainstay of treatment because antibiotics lacked efficacy, and several antiviral agents were applied with unsatisfactory results.

Since the SARS-CoV-2 outbreak began, a series of medical interventions derived from in vitro and in vivo models of other infections has been administered to patients with COVID-19, including lopinavir/ritonavir and 4-aminoquinoline-based combinations, such as chloroquine or hydroxychloroquine.

The first 'hit' of these therapies occurred in March from a randomised, controlled, open-label trial of lopinavir/ritonavir in COVID-19 adults by Cao et al. [3] in which no benefit was observed beyond the standards of care in the treated group. Shortly after, an analysis by Mehra et al. [4] in May associated chloroquine/hydroxychloroquine regimens with decreased in-hospital survival and a higher risk of arrhythmia, supporting the established two-hit hypothesis and seemingly discounting new therapeutic schemes using these drugs. Nevertheless, Mehra et al. unnervingly retracted their ground-breaking article on 5th June, for it was no longer able to ensure the veracity of their primary data sources. It is also worth noting that another very recent original article, entitled 'Cardiovascular disease, drug therapy, and mortality in COVID-19', had appeared in the NEJM but was retracted a few days later for the same reason. This paper had confuted previous concerns regarding the potentially harmful association of ACE inhibitors or ARBs with in-hospital deaths in COVID-19 subjects.

However, these were not the first backtracking steps on therapy and supportive care by elite journals and the academic community during this pandemic. In mid-March, even the World Health Organisation saw fit to retract its earlier warnings against the use of ibuprofen and other non-steroidal anti-inflammatory drugs, which it released a few weeks earlier in response to advice published in the British Medical Journal [5].

These examples of highly public retractions might suggest a lack of rigour in a rush to publish, which has affected some of the most prestigious medical journals.

As a result of this uncertainty, it is likely that many patients have been treated with ineffective first-line drugs or that arguably useful second-line drugs, such as remdesivir, tocilizumab, anakinra, and baricitinib, have been challenging to obtain in many countries.
As physicians, what we are most interested in is treating our patients to the best of our knowledge and sending them home to their families. For these reasons, a lack of treatment safety data and efficacy and times of ambiguity should perhaps demand the rule, 'If uncertain, do not try this at home'.

Author contributions Each author contributed equally to this manuscript.

Funding None.

Availability of data and material Not requested.

\section{Compliance with ethical standards}

Conflict of interest The author(s) declare that they have no conflict of interest.

Statement of human and animal rights This article does not contain any studies with human participants or animals performed by any of the authors.

Informed consent None.

\section{References}

1. Li Q, Guan X, Wu P et al (2020) Early transmission dynamics in Wuhan, China, of novel coronavirus-infected pneumonia. N Engl J Med 382(13):1199-1207. https://doi.org/10.1056/NEJMoa2001 316

2. Zhang R, Li Y, Zhang AL, Wang Y, Molina MJ (2020) Identifying airborne transmission as the dominant route for the spread of COVID-19. Proc Natl Acad Sci USA. https://doi.org/10.1073/ pnas. 2009637117

3. Cao B, Wang Y, Wen D et al (2020) A trial of lopinavir-ritonavir in adults hospitalized with severe Covid-19. N Engl J Med 382(19):1787-1799. https://doi.org/10.1056/nejmoa2001282

4. Mehra MR, Desai SS, Ruschitzka F, Patel AN (2020) Hydroxychloroquine or chloroquine with or without a macrolide for treatment of COVID-19: a multinational registry analysis. The Lancet. https://doi.org/10.1016/s0140-6736(20)31180-6

5. Day M (2020) Covid-19: ibuprofen should not be used for managing symptoms, say doctors and scientists. BMJ 368:1086

Publisher's Note Springer Nature remains neutral with regard to jurisdictional claims in published maps and institutional affiliations. 